\title{
Las políticas públicas en materia de discapacidad en América Latina y su garantía de acceso a una educación inclusiva de calidad
}

Public policies on disability in Latin America and its guarantee of access to quality inclusive education

\section{Volumen 19, Número 2 \\ Mayo-Agosto \\ pp. 1-26}

\section{Raudin Esteban Meléndez Rojas}

\section{Cite este documento así}

Meléndez Rojas, Raudin Esteban. (2019). Las políticas públicas en materia de discapacidad en América Latina y su garantía de acceso a una educación inclusiva de calidad. Revista Actualidades Investigativas en Educación, 19(2), 1-26. Doi. 10.15517/aie.v19i2.36916 


\title{
Las políticas públicas en materia de discapacidad en América Latina y su garantía de acceso a una educación inclusiva de calidad
}

Public policies on disability in Latin America and its guarantee of access to quality inclusive education

\section{Raudin Esteban Meléndez Rojas ${ }^{1}$}

\begin{abstract}
Resumen: Los países de América Latina han dado importantes pasos en materia jurídica e institucional para proteger y promover los derechos y la inclusión social de las personas con discapacidad, tanto a nivel nacional como regional e internacional. Sin embargo, a la par de este avance, los resultados prácticos de la acción de las instituciones y de las políticas públicas implementadas aún requieren del monitoreo y la evaluación rigurosa que señale qué está siendo efectivo. Desde esta perspectiva, este ensayo se plantea dos objetivos, el primero, describir el marco jurídico e institucional de la región para las políticas dirigidas a las personas con discapacidad. El segundo, realizar un breve análisis de estas políticas, con énfasis en la efectividad en el acceso a la educación de este grupo poblacional. Una conclusión derivada del análisis es que mejorar la información, la focalización y la articulación institucional de las políticas resulta clave para alcanzar su efectividad. También se concluye que es necesario plantear una agenda de investigación universitaria en América Latina, con relación a los temas y la problemática que impacta en la protección y promoción de los derechos y la inclusión social de las personas con discapacidad.
\end{abstract}

Palabras clave: accesibilidad, discapacidad, educación, políticas públicas.

\begin{abstract}
Latin American countries have taken important steps in legal and institutional matters to protect and promote the rights and social inclusion of people with disabilities, at the national, regional and international levels. Even though there has been progress, the practical results of the institutions' actions and the implemented public policies still require monitoring and a rigorous evaluation in order to determine if the policies have been effective. From this perspective, this essay proposed two objectives, the first, describes the legal and institutional framework of the region for the policies directed towards people with disabilities. The second, to perform a brief analysis of these policies, with a focus on the effectiveness of access to education for this population group. . A conclusion of this analysis is that the development of better information, a continuing focus, and the institutional articulation of the policies are key for achieving effectiveness in this endeavor. It is also concluded that it is necessary to establish a university research agenda in Latin America, in relation to the issues and problems that impact on the protection and promotion of rights and social inclusion of people with disabilities.
\end{abstract}

Keywords: accessibility, disability, education, public policies.

\footnotetext{
${ }^{1}$ Docente e Investigador de la Universidad de Costa Rica en la Escuela de Administración Pública, Costa Rica. Doctor en Educación por la Universidad Católica de Costa Rica.
}

Dirección electrónica: raudincr@gmail.com

Artículo recibido: 22 de octubre, 2018

Enviado a corrección: 24 de enero, 2019

Aprobado: 29 de abril, 2019 


\section{Introducción}

La discapacidad es un término general "[...] que incluye a personas que tengan deficiencias físicas, mentales, intelectuales o sensoriales a largo plazo que, al interactuar con diversas barreras, pueden impedir su participación plena y efectiva en la sociedad, en igualdad de condiciones con las demás" (Rodríguez y Salazar, 2011, p. 14). Las deficiencias son problemas que afectan una estructura o función corporal; por ejemplo, no oír. Las limitaciones de la actividad son dificultades para ejecutar acciones o tareas; por ejemplo, no poder subir escaleras. Por último, las restricciones de la participación son problemas para participar en situaciones vitales; por ejemplo, no educarse. Según la Organización Mundial de la Salud (OMS), se desprende la noción de que la discapacidad es un fenómeno complejo, multidimensional, que refleja una interacción entre las características del organismo humano y las características de la sociedad en la que se vive (OMS, 2011, p. 3).

Se considera que alrededor de un $15 \%$ de la población mundial, según las estimaciones de esta población en el año 2010, tiene algún tipo de discapacidad. Esta cifra supone un incremento respecto a la medición correspondiente a los años 1970, cuando representaba alrededor del $10 \%$. Este aumento del número absoluto y relativo de personas con alguna discapacidad se debe en gran parte al envejecimiento de la población, pues las personas adultas mayores son muy proclives a sufrir algún tipo de discapacidad (OMS, 2011, pp. 7-8). Entre la amplia gama de discapacidades existentes, las más comunes son: la discapacidad visual, la discapacidad auditiva y del habla y la discapacidad de movilidad. De manera secundaria, pero no menos importante, se observa la discapacidad cognitiva, referida a los problemas relativos a las dificultades para el aprendizaje y la discapacidad psicosocial, relacionada con problemas de conducta. A menudo, una misma persona puede presentar más de una discapacidad (Meléndez Rojas, 2018b, p. 3).

A lo largo de las últimas décadas ha habido un cambio de percepción relevante acerca de las personas con discapacidad, pues se ha pasado de una percepción enfocada en el individuo, en su condición médica, a una que interpreta la discapacidad en su dimensión social, como grupo, y presta atención a las barreras físicas, institucionales y sociales que enfrentan las personas con discapacidad. Se puede hablar entonces de una transición de la visión de las personas con discapacidad, desde un "modelo médico" a un "modelo estructural”, sin que la condición médica y la condición social representen una dicotomía, 
antes, más bien, el nuevo enfoque postula dar un peso relativo apropiado a los diferentes aspectos de la discapacidad (OMS, 2011, p. 4).

El enfoque bio-psicosocial, el cual rompe con el biomédico y asistencialista que existía, ha derivado en una visión contemporizada que hace énfasis en la protección y promoción de los derechos y de la inclusión social de las personas con discapacidad. Por esta razón, en las últimas décadas se ha creado, con el apoyo de instituciones internacionales, regionales y de los gobiernos nacionales, una estructura jurídica y normativa muy completa que refleja este cambio de visión y se corresponde mejor con el diseño e instrumentación de políticas públicas específicas dirigidas a la atención de este grupo de la población. Como consecuencia, en la Organización de Naciones Unidas (ONU) se promovió, desde 2006, la "Convención Internacional sobre los Derechos de las Personas con Discapacidad", la cual está vigente desde mayo del 2008 y ha sido ratificada por 175 países. Con esta Convención se creó un marco normativo supranacional para la promoción, protección y aseguramiento de los derechos humanos y el disfrute de las libertades fundamentales de las personas con discapacidad (ONU, 2006, p. 4).

La Convención ha derivado en que la mayoría de los gobiernos de las naciones adscritos a ella, y con el objeto de cumplir con su mandato, crearán un cuerpo particular de leyes, normativas, instituciones que desarrollarán políticas públicas inherentes, para una atención más efectiva de los derechos y necesidades de las personas con discapacidad. En este sentido, la totalidad de los gobiernos de los países de América Latina ratificaron y se adscribieron a la Convención Internacional sobre los Derechos de las Personas con Discapacidad, a la par que han desarrollado sus propias leyes, normativas, instituciones y políticas, dentro de un enfoque que se corresponde con los principios promovidos y los objetivos buscados de respeto a los derechos humanos y su dignidad, así como el de garantizar su inclusión y participación social.

A pesar que existen discrepancias estadísticas, debido, especialmente, a las diferentes metodologías que se utilizan en los censos poblacionales para determinar la cantidad de personas con discapacidad, de las rondas censales realizadas en 2010 y 2011 se infiere que las personas con alguna discapacidad representan en promedio el 14,2\% de la población latinoamericana. Esto, según los datos de un informe de la Comisión Económica para América Latina (CEPAL, 2013) que incluye 17 países de la región y que sería de $12 \%$ si se toma a América latina en su conjunto. En relación con los datos de varios censos 
poblacionales, González y Stang (2014, pp. 80-89) señalan que la cantidad de personas con alguna discapacidad ha aumentado en porcentaje en la mayoría de los países latinoamericanos, aunque esto se debe en parte al cambio de metodología y de enfoque en las preguntas relacionadas con la discapacidad.

En general, pese a que la mayoría de las naciones latinoamericanas tienen un amplio reconocimiento jurídico de protección de los derechos de las personas con discapacidad, al mismo tiempo que cuentan con instituciones e instrumentan políticas a favor de hacer respetar sus derechos y su inclusión social, en la práctica un importante número de personas sufren de vulnerabilidad social, de una mayor desigualdad de oportunidades y pueden ser sujetos, en mayor grado que otros grupos, de exclusión en el acceso a derechos sociales claves como la educación o el empleo (Stang, 2011).

De acuerdo con la OMS (2011), "[...] las personas con discapacidad presentan tasas más altas de pobreza que las personas sin discapacidad" (p.12). Al respecto, existe una suerte de vínculo bidireccional entre la pobreza y la discapacidad, pues la pobreza incrementa el riesgo de padecer alguna discapacidad y la discapacidad eleva el riesgo de estar dentro de la pobreza. En el mundo entero, las personas con discapacidad suelen tener peores resultados académicos, menores tasas de participación económica y unas tasas de pobreza más altas que las personas sin discapacidad. Todo ello es en gran parte el resultado de los obstáculos que entorpecen y dificultan el acceso de las personas con discapacidad a servicios como la educación, la salud, el transporte, el empleo, la información (OMS, 2011).

En relación con lo anterior, garantizar el acceso a la educación inclusiva y de calidad de las personas con discapacidad, especialmente de niños, niñas y adolescentes con esta condición, es un aspecto clave para derribar las barreras que le impiden a este grupo de la población asegurarse de servicios que son relevantes para su inclusión social, su actualización y su inserción laboral. Desde esta perspectiva, este ensayo se plantea dos objetivos: en primer lugar, describir el marco jurídico e institucional que rige en América Latina en materia de los planes, programas y políticas dirigidas a las personas con discapacidad. En segundo lugar, analizar las políticas públicas de acceso a la educación para las personas con discapacidad de la región.

En este orden de ideas, el presente ensayo se estructura en cinco partes, de las cuales esta introducción es la primera. En la segunda parte se describe someramente el marco jurídico que rige, en general, en América Latina con respecto al aseguramiento de los 
derechos y la promoción de la inclusión social de las personas con discapacidad. En la tercera parte se describe y analiza el marco institucional que materializa, con base en la normativa jurídica, las acciones y el diseño e instrumentación de políticas públicas dirigidas de manera específica o coordinada con otras políticas hacia las personas con discapacidad, haciendo énfasis en las políticas de acceso a la educación. En la cuarta parte se analiza comparativamente, con datos e información, la efectividad de las políticas de acceso a la educación de las personas con discapacidad en la región. En la quinta parte se ventilan algunas consideraciones finales en relación con los desafíos que enfrentan las políticas educativas para las personas con discapacidad en América Latina.

Es importante señalar que este ensayo científico es producto de una investigación doctoral de tipo cualitativo, realizada por el proponente, en la cual se analizó la implementación de las políticas públicas educativas para personas con discapacidad visual en Costa Rica, y como parte del estudio, se realizó una comparación de las políticas públicas de acceso a la educación para las personas con discapacidad en América Latina. Por ende, siguiendo el método de la hermenéutica crítica, se desarrolló este documento que compila una parte importante de esa investigación doctoral.

\section{Marco jurídico referido a las personas con discapacidad en América Latina}

Desde una perspectiva regional, América Latina ha estado adelantada en generar un marco jurídico y normativo muy completo en torno a los derechos, las instituciones y las políticas públicas dirigidas a las personas con discapacidad. En efecto, en 1999 un grupo importante de naciones latinoamericanas ratificaron la "Convención Interamericana para la Eliminación de todas las formas de Discriminación contra las personas con Discapacidad", promovida por la Organización de los Estados Americanos (OEA). Los objetivos de esta Convención fueron prevenir y eliminar todas las formas de discriminación contra las personas con discapacidad e instar a lograr la plena inclusión social mediante políticas y programas sociales y educativos (OEA, 1999).

Posteriormente, en 2006 dentro del seno de la OEA se promulgó la "Declaración del Decenio de las Américas por los Derechos y la Dignidad de las Personas con Discapacidad (2006-2016)". Esta Declaración, ratificada por los Estados miembros, contemplaba un programa de acción que los comprometía a adoptar gradualmente y dentro del plazo de una década medidas legislativas, administrativas, así como políticas públicas necesarias para 
mejorara la inclusión social y la calidad de vida de las personas con discapacidad en aspectos como la salud, educación, empleo, accesibilidad, participación política, cultura, asistencia social y cooperación internacional (Ullmann, 2017, p. 276).

Tal como se señaló, prácticamente la totalidad de las naciones latinoamericanas ratificaron la "Convención Internacional sobre los Derechos de las Personas con Discapacidad". Una primera aproximación al tratamiento de los derechos de las personas con discapacidad puede partir de describir brevemente los aspectos más relevantes de esta Convención. En ella se menciona que las personas con discapacidad gozan plenamente de "las condiciones de igualdad de todos los derechos humanos y libertades fundamentales por todas las personas con discapacidad, y promover el respeto de su dignidad inherente" (ONU, 2006, p. 8). Asimismo, las personas con discapacidad tienen derecho al respeto de su dignidad, a la autonomía individual, a la no discriminación, a la inclusión y la participación en la sociedad, a la igualdad de oportunidades, la accesibilidad, el respeto a la evolución de las facultades de los niños y las niñas con discapacidad y de su derecho a preservar su identidad (ONU, 2006, pp. 4-6). La ratificación de la Convención exige el compromiso de cumplir una serie de obligaciones entre las que se cuentan:

- Adoptar las medidas legislativas, administrativas y de otra índole que sean pertinentes para hacer efectivos los derechos de las personas con discapacidad.

- Tener en cuenta en todas las políticas y en todos los programas, la protección y promoción de los derechos humanos de las personas con discapacidad.

- Tomar las medidas pertinentes para que ninguna persona, organización o empresa privada discrimine por motivos de discapacidad.

- Proporcionar información que sea accesible para las personas con discapacidad sobre ayudas a la movilidad, dispositivos técnicos y otras tecnologías de apoyo.

- Promover la formación de profesionales y del recurso humano que trabajan con las personas con discapacidad, a fin de mejorar la asistencia y los servicios brindados.

- Adoptar las medidas, en cuanto a recursos disponibles, para asegurar el cumplimiento de los derechos económicos, sociales y culturales de las personas con discapacidad (ONU, 2006, pp. 6-7). 
En relación con el derecho de las personas con discapacidad a tener acceso a la educación, la Convención incorpora una serie de mandatos orientados a promoverlo y asegurarlo. Estos derechos, que también lo son de igualdad de oportunidades, se expresan del siguiente modo:

- Desarrollar el máximo potencial de personalidad, talento y creatividad que tienen las personas con discapacidad, así como sus aptitudes mentales y físicas.

- Asegurar que las personas con discapacidad no queden excluidas del sistema general de educación por este motivo.

- Garantizar que niños, niñas y adolescentes con discapacidad no queden excluidos de su escolaridad primaria y secundaria, brindada de forma gratuita, dentro de su comunidad.

- Prestar el apoyo necesario a las personas con discapacidad, en el marco del sistema general de educación, para facilitar su formación efectiva.

- Facilitar medidas de apoyo personalizadas y efectivas en entornos que fomenten al máximo su desarrollo académico y social (ONU, 2006, pp. 19-20).

La adhesión a los instrumentos jurídicos y normativos internacionales y regionales en materia de derechos y políticas para las personas con discapacidad se ha complementado con la creación de instrumentos legislativos propios de cada país latinoamericano. Por esta razón, la mayoría de los países de la región cuentan con leyes, normativas y disposiciones particulares, las cuales refuerzan esos derechos, a la vez que otorgan una base jurídica a las instituciones responsables de las políticas públicas en esta materia.

Desde la perspectiva de los derechos, instituciones y políticas para las personas con discapacidad, Stang (2011, p. 30) observa que para 2010 había 17 naciones latinoamericanas con, al menos, un artículo en sus constituciones referido a esta población. Por su parte, Heidi Ullmann (2017, p. 278) sostiene que además de los artículos constitucionales que hacen mención explícita y específica de las personas con discapacidad, existen otros artículos de índole jurídica que también contemplan a este grupo de la población, considerado dentro de los grupos sociales vulnerables. Para esta misma autora, existen, además de las legislaciones nacionales específicas en materia de garantizar, proteger y promover los derechos y la inclusión social de las personas con discapacidad, un cuerpo de leyes, decretos, disposiciones y regulaciones que también los abarca, como los 
códigos de la niñez, del trabajo, de la familia, entre otros. Algunas de las leyes, los decretos y las disposiciones nacionales en materia de discapacidad tienen un largo tiempo de haberse promulgado. La más antigua es la Ley del Sistema de Protección Integral de los Discapacitados de Argentina, sancionada en 1981. No obstante, estos instrumentos jurídicos y normativos específicos de la discapacidad se han ido renovando y evolucionando en cuanto a su articulado, en la medida que ha cambiado la percepción en la región hacia las personas con discapacidad, las cuales, como se dijo, ahora no son vistas desde la dimensión biomédica exclusivamente, sino también desde una perspectiva bio-psicosocial

Como lo documenta Ullmann (2017, p. 279), en la década de 1990, seis países aprobaron sus respectivas legislaciones particulares en el ámbito de la discapacidad, mientras que desde la década del año 2000, se experimentó un importante incremento en la región en el número de leyes y decretos nacionales orientados a promover, proteger y garantizar los derechos, la inclusión social y la participación de las personas con discapacidad.

En la Tabla 1 se presentan las leyes y decretos dirigidos, específicamente, al grupo de población conformado por las personas con discapacidad, promulgados en un grupo de 16 países latinoamericanos. La selección se corresponde con una muestra aleatoria, aunque suficientemente representativa, de países de Norteamérica, Centroamérica, Suramérica y el Caribe. La información sobre las diferentes legislaciones es un recurso público disponible en páginas web oficiales. 
Tabla 1

Leyes y Decretos específicos sobre personas con discapacidad para 16 países latinoamericanos

\begin{tabular}{|c|c|c|}
\hline País & Ley o Decreto & $\begin{array}{l}\text { Fecha de } \\
\text { Promulgación }\end{array}$ \\
\hline Argentina & 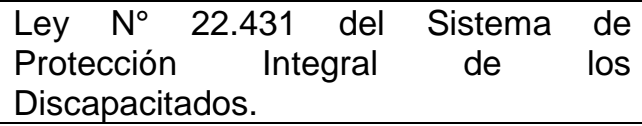 & 1981 \\
\hline Bolivia & $\begin{array}{l}\text { Ley } N^{\circ} 223 \text {. Ley General para personas } \\
\text { con Discapacidad. }\end{array}$ & 2012 \\
\hline Brasil & $\begin{array}{l}\text { Ley } N^{\circ} 7.583 \text { sobre el Apoyo a las } \\
\text { Personas con Discapacidad y su } \\
\text { Integración Social. }\end{array}$ & 1989 \\
\hline Chile & $\begin{array}{l}\text { Ley } \mathrm{N}^{\circ} 20.422 \text { de Normas sobre } \\
\text { lgualdad de Oportunidades e Inclusión } \\
\text { Social de Personas con Discapacidad. }\end{array}$ & 2010 \\
\hline Colombia & $\begin{array}{l}\text { Ley Estatutaria } 1.618 \text { de Disposiciones } \\
\text { para garantizar el pleno ejercicio de los } \\
\text { derechos de las personas con } \\
\text { discapacidad. }\end{array}$ & 2013 \\
\hline Costa Rica & $\begin{array}{l}\text { Ley } \mathrm{N}^{\circ} 7.600 \text { de lgualdad } \mathrm{de} \\
\text { Oportunidades para Personas con } \\
\text { Discapacidad. }\end{array}$ & 1996 \\
\hline Ecuador & Ley Orgánica de Discapacidades. & 2012 \\
\hline El Salvador & $\begin{array}{l}\text { Decreto } N^{\circ} 888 \text {, Ley de Equiparación } \\
\text { de Oportunidades para las personas } \\
\text { con Discapacidad. }\end{array}$ & 2000 \\
\hline Guatemala & $\begin{array}{l}\text { Decreto } N^{\circ} 135-96 \text {, Ley de Atención a } \\
\text { las Personas con Discapacidad. }\end{array}$ & 1997 \\
\hline México & $\begin{array}{l}\text { Ley General de las personas con } \\
\text { Discapacidad. }\end{array}$ & 2005 \\
\hline Nicaragua & $\begin{array}{l}\text { Ley } N^{\circ} 763 \text { de los Derechos de las } \\
\text { Personas con Discapacidad. }\end{array}$ & 2011 \\
\hline Panamá & $\begin{array}{l}\text { Ley } \mathrm{N}^{\circ} 42 \text { de Equiparación de } \\
\text { Oportunidades para las Personas con } \\
\text { Discapacidad. }\end{array}$ & 1999 \\
\hline Perú & $\begin{array}{l}\text { Ley } \mathrm{N}^{\circ} \text { 29.973. Ley General de la } \\
\text { Persona con Discapacidad. }\end{array}$ & 2012 \\
\hline $\begin{array}{l}\text { República } \\
\text { Dominicana }\end{array}$ & $\begin{array}{l}\text { Ley } \mathrm{N}^{\circ} 5-13 \text { Sobre Personas con } \\
\text { Discapacidad. }\end{array}$ & 2013 \\
\hline Uruguay & $\begin{array}{l}\text { Ley } N^{\circ} 18.651 \text { de protección Integral de } \\
\text { personas con Discapacidad. }\end{array}$ & 2010 \\
\hline Venezuela & $\begin{array}{lccc}\text { Ley para } & \text { las } & \text { Personas } & \text { con } \\
\text { Discapacidad. } & & & \\
\end{array}$ & 2006 \\
\hline
\end{tabular}

Fuente: Elaboración propia a partir de la información jurídica de cada país. 
De esta breve descripción del ámbito jurídico y normativo respecto a la protección y promoción de los derechos de las personas con discapacidad en América Latina, se puede destacar, como lo sostiene Ullmann (2017, p. 280), que existe un importante respaldo en la normativa jurídica, a nivel internacional, regional y nacional, hacia esos derechos. De esa normativa jurídica se deriva una visión holística e integral de la discapacidad, reflejada en instituciones gubernamentales que la materializan y en acciones de políticas públicas enmarcadas en esta visión. Por lo demás, este marco jurídico confiere estabilidad y permanencia al abordaje de esta temática, al mismo tiempo que expresa el compromiso del Estado por promover y proteger los derechos y la inclusión social de las personas con discapacidad.

En este sentido, para hacer cumplir e instrumentar, en la práctica, el articulado correspondiente a las leyes que garantizan y protegen los derechos de las personas con discapacidad en América Latina, la mayoría de los gobiernos han creado, nuevamente mediante leyes, decretos y disposiciones, instituciones y organismos ad hoc, con el objeto de coadyuvar en el cumplimiento de las leyes para las personas con discapacidad y diseñar e instrumentar las políticas públicas necesarias para dar tal cumplimiento. En el próximo apartado se analiza brevemente esta vinculación, especialmente la referida a las políticas de acceso a una educación inclusiva y de calidad.

\section{Marco institucional y de políticas públicas referido a las personas con discapacidad en América Latina}

En la totalidad de los países de América Latina existen instituciones con instrumentos legislativos y de política pública específicos, abocadas a hacer cumplir los derechos de las personas con discapacidad y a diseñar e implementar políticas públicas que materialicen la inclusión y participación social plena de este grupo poblacional. Para ello, dichas instituciones responden a sus mandatos legales y ejecutan una serie de acciones y estrategias tendientes a lograr las metas propuestas. Algunas de estas instituciones fueron creadas y respaldadas con leyes anteriores a la promulgación del código legislativo más amplio que rige la materia en el país. Por ejemplo, en 1973, se creó, en Costa Rica, mediante Decreto-Ley, el Consejo Nacional de Rehabilitación y Educación Especial (CNREE), convirtiéndose posteriormente en el Consejo Nacional de Personas con Discapacidad (CONAPDIS) en 2015 (Meléndez Rojas, 2018b, p. 11). Dichas instituciones 
experimentaron un aumento significativo desde la primera década del siglo XXI y en especial a raíz de que los países de la región ratificaron la Convención Regional de la OEA y la Convención Internacional de la ONU en materia de derechos y acciones para las personas con discapacidad.

Las instituciones de carácter público requieren de un presupuesto para su funcionamiento operativo y para el alcance de sus objetivos. Al respecto, Meléndez Rojas (2018a) señala que en el presupuesto público "se contempla el manejo de los recursos financieros de una organización durante el ejercicio anual, mediante una estructura de ingreso-gasto. Es un instrumento de gestión, y se define como la expresión financiera del PAO (Plan Anual Operativo)" (p.37).

Los presupuestos públicos de estas instituciones, como los de la mayoría de los programas de desarrollo social, a menudo están sometidos a las volatilidades fiscales que pueden sufrir los países de la región. Algunas de estas instituciones dependen de un ministerio del ámbito del desarrollo económico y social, que se encarga de formular los lineamientos y políticas de estas instituciones superiores, o en su defecto, dichas organizaciones pueden tener una relativa autonomía de decisión y acción en la definición de sus políticas y estrategias en el cumplimiento de sus objetivos. En algunos casos, se trata de instituciones centralizadas, mientras que en otros están descentralizadas, las cuales coordinan sus acciones con las instancias locales de la propia institución y otros organismos de desarrollo social local. En la mayoría de los casos estas instituciones son responsables de guiar el trabajo de coordinación y de articulación necesario a nivel intersectorial e interinstitucional (Ullmann, 2017).

En general, las principales instancias del gobierno encargadas de coordinar la atención a las personas con discapacidad en la región son, en orden del mayor número de instituciones, los ministerios de desarrollo social, los ministerios de otras carteras y la presidencia ejecutiva (Martínez y Maldonado Valera, 2017).

Además de las instituciones públicas que atienden la protección de los derechos y otras necesidades de las personas con discapacidad, existe en un gran número de naciones latinoamericanas asociaciones y organizaciones no gubernamentales (ONG) que prestan servicios de apoyo en esta materia. La mayoría de estas asociaciones y ONG se encuentran agrupadas en la Red Latinoamericana de Organizaciones No Gubernamentales de Personas con Discapacidad y sus Familias (RIADIS). Creada en 2002, según sus estatutos, esta 
Red promueve y protege los derechos de las personas con discapacidad en América Latina y el Caribe, alentando la no discriminación en la sociedad y promoviendo su desarrollo incluyente por la mejoría de su calidad de vida (RIADIS, 2018).

De acuerdo con Meléndez Rojas (2018a, pp. 37-40), las instituciones y organizaciones públicas dedicadas a instrumentar políticas y estrategias en torno a las personas con discapacidad se enmarcan dentro de los llamados desarrollos institucionales focalizados, los cuales tienen por mandato garantizar derechos de ciertos segmentos poblacionales como los afrodescendientes, niños, niñas y adolescentes, las etnias indígenas y los migrantes, cuyas características hacen necesaria una especialización de la acción pública. Sin embargo, dado que las personas con discapacidad conforman un colectivo transversal, y de cuyos derechos, protección y necesidades se encargan diferentes organismos públicos, algunas de estas políticas y estrategias se yuxtaponen o se complementan. Por poner un ejemplo en este contexto, en Paraguay los programas de transferencia condicionada de la Secretaría de la Presidencia de Acción Social (SAS) se coordinan con la Secretaría Nacional por los Derechos de las Personas con Discapacidad (SENADIS), esto para garantizar una transferencia monetaria a las familias en las que haya una persona con una discapacidad severa, con el fin de ayudar en su cuidado (Rico y Robles, 2017, p. 237).

Cuando una problemática afecta a la población de personas con discapacidad, la articulación e integración de determinadas políticas cobra gran relevancia. Como lo sostiene Nazif (2011), para el caso de las políticas relacionadas con los servicios de transporte y las vinculadas con la movilidad de las personas con discapacidad, estas deben abordarse en alguna medida de manera integral, coordinada, pues solo así se pueden esperar resultados efectivos. Al respecto, el autor pone el ejemplo de las políticas de transporte y de movilidad. En general, América Latina es una región donde los servicios de transporte no consideran las condiciones particulares y específicas de movilidad de las personas con discapacidad. Por tanto, se deben fomentar aquellas políticas de servicios de transporte que tengan un diseño y una operatividad que sea inclusiva e integradora. Para lograrlo se pueden proponer una serie de lineamientos institucionales y técnicos que apunten a una interacción virtuosa de las dos políticas, en procura de la movilidad de las personas con discapacidad.

Por lo demás, en casi todos los países de la región existe un plan, estrategia o política como instrumento para guiar las acciones en materia de discapacidad, sea articulado con otras instancias institucionales, sea centralizado en la propia institución responsable de las 
políticas para las personas con discapacidad. En estos instrumentos, con frecuencia, se explicitan los mecanismos para su implementación, ejecución, monitoreo y evaluación, al establecer líneas de acción, actividades, plazos de ejecución, asignación de roles a los diferentes actores sociales y temporalidad de las acciones. No obstante, son pocos los países que cuentan con un sistema de información y de indicadores que permita dar seguimiento a estas políticas públicas (Ullmann, 2017, p. 293).

En lo que respecta al derecho particular de tener acceso a la educación, el fin de las políticas públicas relacionadas con garantizarlo para las personas con discapacidad, especialmente para niños, niñas y adolescentes, está vinculado con el Objetivo de Desarrollo Sostenible número cuatro (ODS-4) formulado por el Programa de Naciones Unidas para el Desarrollo (PNUD). El ODS-4 se plantea garantizar una educación inclusiva y equitativa de calidad promoviendo oportunidades de aprendizaje permanente para todos. Una de sus metas al 2030 es explícita en plantear que para esa fecha deben haberse eliminado las disparidades de género en la educación, garantizado el acceso a las personas vulnerables y asegurado la inclusión de las personas con discapacidad (PNUD, 2015).

Pese a los buenos propósitos enunciados en el ODS-4, actualmente el acceso a la educación de las personas con discapacidad en buena parte de los países de América Latina confronta obstáculos y dificultades. Colamarco y Delamonica (2013) señalan que la falta de inclusión educativa de los niños, las niñas y adolescentes con alguna discapacidad no solo violenta sus derechos fundamentales, sino también perjudica a la sociedad a corto y a largo plazo.

Entre estos obstáculos se señala que las personas con discapacidad están sobrerepresentadas en la tasa de pobreza del país, es decir, un alto porcentaje relativo de ellas son pobres y esto termina siendo una limitación relevante para el acceso a la educación. A pesar de la existencia de las políticas lo que dificulta el acceso a la educación es el contexto. A menudo, las instituciones educativas no tienen la infraestructura adecuada para su movilidad, no cuentan o no tienen las condiciones adecuadas para el uso de dispositivos tecnológicos apropiados para las diferentes discapacidades, ni cuentan con personal docente suficientemente capacitado o con las competencias para la atención del estudiantado con discapacidad.

Por esta razón, las políticas educativas dirigidas a asegurar la inclusión también deben contemplar garantizar la calidad de la educación. Como lo sostienen Bernal y Gómez (2012, 
p. 50), la educación inclusiva de calidad ayuda a neutralizar la brecha desfavorable de aprendizaje del estudiantado con alguna discapacidad de los que no tienen esta condición. Lograr cerrar esta brecha supone acciones afirmativas como apoyo escolar focalizado, intensivo y constante para mejorar el aprendizaje de esta población con alguna discapacidad y la aplicación de estrategias pedagógicas que fomenten un mejor aprendizaje para todos.

Por su parte, las políticas dirigidas a mejorar la infraestructura educativa para adaptarla al tipo de movilidad que tienen las personas con discapacidad y las orientadas a la dotación de dispositivos tecnológicos apropiados y competentes para la labor de enseñanza y aprendizaje, también coadyuvan a mejorar el proceso educativo. Adicionalmente, la formación continua del personal docente, así como la vinculación de la familia e incluso de la comunidad en el proceso educativo, pueden resultar claves para obtener buenos resultados al respecto.

La existencia de estas políticas para enfrentar los obstáculos y limitaciones que impone la condición de discapacidad en la niñez y la adolescencia requiere de una buena coordinación y articulación interinstitucional para solventarlos. Por poner un ejemplo de la relevancia de ello, aunque en Costa Rica la institución rectora de las políticas para las personas con discapacidad es el Consejo Nacional de Personas con Discapacidad (CONAPDIS) adscrito al Ministerio del Trabajo, los lineamientos de la política educativa inclusiva y de calidad, dirigidos a niños, niñas y adolescentes con alguna discapacidad que asisten a escuelas regulares o centros especializados, son establecidos y coordinados con estas instituciones por el Ministerio de Educación Pública (Meléndez Rojas, 2018b).

En relación con lo anterior, la gama de políticas diseñadas e implementadas no implica que las escuelas para niños, niñas y adolescentes con discapacidad deban ser uniformes. En realidad, las políticas públicas al respecto se sirven como mínimo de tres modelos de escuela. El primer modelo es el de las escuelas especiales, dependiendo del tipo de discapacidad, escuelas integradas con clases especiales para estudiantes con discapacidad y escuelas inclusivas, donde estudiantes con discapacidad estudian y reciben todas sus clases junto con estudiantes sin discapacidad. De lo que se trata en todas ellas es de asegurar la igualdad de oportunidades en su proceso de enseñanza y aprendizaje.

Dado que el acceso a una educación inclusiva y de calidad se fundamenta en la capacidad natural que tiene toda persona de aprender, las políticas educativas específicas para la niñez con alguna discapacidad deben coordinarse con las políticas educativas 
basadas en un currículo flexible y métodos de enseñanza y aprendizaje adaptados a las diferentes fortalezas y estilos de aprendizaje estudiantil. Como un ejemplo de ello, Meléndez Rojas (2018b, p. 21) señala que en el caso de Costa Rica, tanto en los institutos de educación especial para la niñez con alguna discapacidad, así como en las escuelas públicas donde están integrados, se siguen los lineamientos de política educativa dictados por el Ministerio de Educación Pública, los cuales garantizan una educación para todos basada en el humanismo, el racionalismo y el socio-constructivismo.

En este orden de ideas, como lo sostienen Colamarco y Delamonica (2013), algunos países de América Latina entienden el proceso de brindar una educación inclusiva no solo como una filosofía o un enfoque educativo, sino también como un asunto de política pública, donde es fundamental garantizar el derecho a la educación a la par de ofrecer una educación de calidad. A pesar de lo anterior, en lo concerniente a la necesaria coordinación interinstitucional eficiente para lograr la efectividad de las políticas educativas, especialmente las dirigidas a las personas con discapacidad, la mayoría de los países de la región están rezagados en esta materia. Las deficiencias en la coordinación interinstitucional se acentúan debido a la precariedad o inexistencia de sistemas estadísticos, sistemas de información y de indicadores que permitan monitorear y evaluar las políticas implementadas. Esta limitación impide hacer un análisis más pertinente de la efectividad de las políticas educativas, aunque en varios países de la región están documentados una serie de estudios de caso exitosos referidos a estas políticas (Bernal y Gómez, 2012; Rico y Robles, 2017). Como se observa, las políticas públicas en materia de discapacidad no garantizan por sí solas el acceso a una educación inclusiva de calidad. Se pueden ver entorpecidas por el contexto, por una mediación pedagógica poco flexible que no contempla fortalezas y estilos de aprendizaje de los estudiantes, por la poca información que se tiene y por la falta de coordinación institucional.

En consonancia con lo expuesto, en el apartado cuatro se va a realizar un análisis de las políticas educativas para las personas con discapacidad basado en algunos datos estadísticos existentes sobre el acceso a la educación primaria y secundaria de niños, niñas y adolescentes con alguna discapacidad. Este análisis arrojará resultados comparativos en cuanto a la efectividad de la política educativa inclusiva, aunque no dice nada acerca de la calidad educativa. 
4. Análisis comparativo de la efectividad de la política de acceso a la educación para la niñez y adolescencia con discapacidad en América Latina

En la introducción de este trabajo se mencionó que las estadísticas más actualizadas sobre el grupo de población que conforman las personas con discapacidad en la mayoría de los países de América Latina dependen de la metodología utilizada para definir la discapacidad, de acuerdo a cómo se abordó esta definición y su aplicación en las rondas censales de 2010 y 2011 en la región.

En general, se tiene la percepción de que los datos sobre personas con discapacidad están en alguna medida subestimados, en especial, en aquellos países donde los censos no incluyeron preguntas perfectamente enfocadas a determinar la prevalencia de la discapacidad en las encuestas sociodemográficas dirigidas a los hogares. Tomando en cuenta estas limitaciones y contando con los datos de CEPAL (2013) sobre prevalencia de la discapacidad, que incluye 17 países de la región, se realizaron algunas estimaciones propias y se presenta la información pertinente en la Tabla 2 para objeto de análisis.

Tabla 2

Porcentaje de la población total con discapacidad, por país según sexo en el periodo 2012

\begin{tabular}{|l|c|c|c|}
\hline País & Hombres & Mujeres & Total \\
\hline Argentina & 6,8 & 7,3 & 7,1 \\
\hline Brasil & 21,2 & 26,5 & 23,9 \\
\hline Chile & 10,9 & 14,9 & 12,9 \\
\hline Colombia & 6,6 & 6,3 & 6,5 \\
\hline Costa Rica & 10,4 & 10,7 & 10,6 \\
\hline Ecuador & 6,6 & 5,6 & 6,1 \\
\hline El Salvador & 4,5 & 3,7 & 4,1 \\
\hline Guatemala & 3,6 & 3,2 & 3,4 \\
\hline Haití & 1,5 & 1,5 & 1,5 \\
\hline Honduras & 3 & 2,3 & 2,7 \\
\hline México & 5,2 & 5,1 & 5,2 \\
\hline Panamá & 3,3 & 3 & 3,2 \\
\hline Paraguay & 1,1 & 0,9 & 1,0 \\
\hline Perú & 7,6 & 9,2 & 8,4 \\
\hline República Dominicana & 4,3 & 4,1 & 4,2 \\
\hline Uruguay & 13,8 & 18,7 & 16,3 \\
\hline Venezuela & 4,1 & 3,8 & 4,0 \\
\hline América Latina-17 & & & 14,2 \\
\hline
\end{tabular}

Fuente: Elaboración propia con datos de CEPAL (2013, p. 202). 
Aunque la región en su conjunto presenta una tasa de personas con discapacidad en promedio de $14,2 \%$ del total de la población, se observan diferencias en las tasas de personas con discapacidad cuando se toman las naciones latinoamericanas de manera individual. Mientras las tasas más altas de personas con discapacidad se encuentran en Brasil, de $23,9 \%$ del total de la población, y Uruguay (16,3\%), las respectivas tasas más bajas corresponden a Paraguay, de apenas $1 \%$ del total de la población, y Haití (1,5\%). El país que tiene la tasa de personas con discapacidad más cercana al promedio latinoamericano es Chile, de 12,9\% del total de la población.

A pesar de las diferencias porcentuales antes mencionadas, se observa que los países de la región guardan más semejanzas. De los datos de la Tabla 2 se puede observar que, en seis países, la tasa de personas con discapacidad es mayor entre las mujeres que la respectiva para los hombres; en otro grupo no se destacan diferencias apreciables; mientras que en un tercer grupo la tasa de discapacidad es mayor entre los hombres. La mayor expectativa de vida, en promedio, de las mujeres y la mayor tasa de envejecimiento que exhiben naciones como Uruguay, Chile, Argentina y Brasil, parece ser una explicación plausible a por qué el grupo femenino tiene tasas de discapacidad mayor.

Aunque no se muestra en los datos de la Tabla 2, pero sí se refleja en el informe de la CEPAL (2013), en todos los países, sin excepción, la tasa de discapacidad aumenta conforme aumenta el tramo de edad que se considere. El tramo de menor edad es de 0-4 años y el tramo de mayor edad es de 60 años o más años.

La presencia de alguna discapacidad se agudiza en la edad adulta (de 40 a 59 años) y se acentúa mucho más en las personas mayores de 60 años, que es el grupo de edad donde se observa la mayor tasa de personas con discapacidad en todos los países de la región. La tendencia hacia el incremento del porcentaje de población mayor a 60 años que exhibe la región es un indicativo de que la tasa de personas con discapacidad probablemente también aumentará.

Otro aspecto donde son bastante similares las estadísticas sobre discapacidad en la región es en lo relativo al tipo de discapacidad más frecuente. En este sentido, las discapacidades más recurrentes son, en este orden, la discapacidad visual (cualquier tipo de deficiencia visual, excepto el uso de anteojos), la discapacidad en la movilidad (limitaciones para caminar, subir escaleras o mover las extremidades) y la discapacidad auditiva (deficiencias de audición y de habla). 
Por su parte, en un grupo de siete países estudiados (Colombia, Costa Rica, Ecuador, El Salvador, México, Paraguay, Uruguay) se determinó que la prevalencia de la discapacidad se manifiesta más en el medio rural que en el medio urbano. Para otro grupo de países de la región (Brasil, Colombia, Costa Rica, Ecuador, El Salvador, México, Panamá, Uruguay) se estableció que la condición étnica influye en la tasa de prevalencia de discapacidad, pues representa una tasa más alta entre los afrodescendientes y los indígenas, aunque conforme suben los tramos de edad, la brecha se cierra en alguna medida. En cuanto a la relación entre discapacidad y nivel de ingresos, la información para tres países: Chile, Costa Rica y México deja en claro que la tasa de discapacidad es más alta en los hogares con los niveles de ingreso más bajos, en los hogares pobres, y los problemas atinentes a la falta de atención de la discapacidad se agudizan conforme las personas con discapacidad de esos hogares pobres envejecen (CEPAL, 2013).

Con el propósito de cumplir con uno de los objetivos puntuales de este ensayo, se realizó, en primer lugar, una descripción de los porcentajes que representan los niños, las niñas y adolescentes con discapacidad, particularmente los del grupo de edad comprendido entre 0 a 19 años, en el total de la población de 17 países de la región, según los datos contenidos en CEPAL (2013).

Al respecto, las personas con discapacidad en la edad comprendida entre 0 y 4 años del género masculino es $2 \%$, mientras que el respectivo para el femenino es $1,6 \%$. Este porcentaje se eleva para el grupo de edad comprendido entre 5 y 12 años, hasta 3,7\% para los niños y $3,1 \%$ para las niñas. Las tasas respectivas para las edades comprendidas entre 13 y 19 años son 3,7\% para los hombres y 3,4\% para las mujeres. Se puede inferir que la tasa de prevalencia de la discapacidad entre niños, niñas y adolescentes en edad escolar oscila entre 3 y $4 \%$ en promedio para 17 países de la región.

En segundo lugar, se presentan datos de la tasa neta de matrícula en el primer y segundo nivel de enseñanza, para la edad escolar de las niñas, los niños y adolescentes para 8 países seleccionados de la región. La selección se realizó atendiendo al criterio de que, además de la información estadística pertinente sobre estas tasas, el país también contara con información estadística de la tasa de escolaridad para el grupo transversal que conforman las personas con discapacidad, con el objeto de hacer la respectiva comparación. En este sentido, en la Tabla 3 se exponen los datos estadísticos de las tasas de matrícula educativa, mientras que en la Tabla 4 se refleja, para los mismos países, los respectivos de 
las personas con discapacidad de edades entre 13 y 18 años, por tipo de discapacidad más recurrente (visual, auditiva, del habla, del aprendizaje, de conducta, de movilidad).

Tabla 3

Porcentaje de matrícula, por país según nivel de enseñanza en diversos periodos

\begin{tabular}{|l|c|c|}
\hline País (año del dato) & $\begin{array}{l}\text { Tasa } \\
\text { Matrícula } \mathbf{1}^{\circ} \text { Nivel }\end{array}$ & $\begin{array}{l}\text { Tasa } \\
\text { Matrícula 2 }{ }^{\circ} \text { Nivel }\end{array}$ \\
\hline Brasil (2011) & 95 & 78 \\
\hline Colombia (2005) & 94 & 64 \\
\hline Costa Rica (2011) & 98 & 76 \\
\hline Ecuador (2010) & 95 & 73 \\
\hline El Salvador (2007) & 94 & 57 \\
\hline México (2010) & 96 & 68 \\
\hline Panamá (2010) & 96 & 66 \\
\hline Uruguay (2010) & 100 & 72 \\
\hline
\end{tabular}

Fuente: Elaboración propia con datos de CEPALSTAT (CEPAL, 2018).

Tabla 4

Porcentaje de escolaridad del total de personas de 13-18 años, por tipo de discapacidad según país en diversos periodos

\begin{tabular}{|c|c|c|c|c|c|c|c|c|}
\hline Discapacidad/País & $\begin{array}{c}\text { Brasil } \\
(2010)\end{array}$ & $\begin{array}{c}\text { Colombia } \\
(2005)\end{array}$ & $\begin{array}{c}\text { Costa } \\
\text { Rica } \\
(2011)\end{array}$ & $\begin{array}{c}\text { Ecuador } \\
(2010)\end{array}$ & $\begin{array}{c}\text { El } \\
\text { Salvador } \\
(2007)\end{array}$ & $\begin{array}{c}\text { México } \\
(2010)\end{array}$ & $\begin{array}{c}\text { Panamá } \\
(2010)\end{array}$ & $\begin{array}{c}\text { Uruguay } \\
(2011)\end{array}$ \\
\hline Visual & 89 & 75 & 88 & 84 & 65 & 80 & 88 & 87 \\
\hline Auditiva & 86 & 59 & 84 & 76 & 44 & 71 & 80 & 84 \\
\hline Del habla & - & 46 & 81 & - & 28 & 61 & 70 & - \\
\hline Del Aprendizaje & - & 47 & 79 & 60 & - & 66 & 79 & 82 \\
\hline De conducta & 68 & 39 & 76 & 63 & 17 & 46 & - & - \\
\hline De movilidad & 74 & 51 & 77 & 71 & 37 & 63 & 67 & 76 \\
\hline Promedio & 79 & 53 & 80 & 71 & 38 & 65 & 77 & 82 \\
\hline
\end{tabular}

Fuente: Elaboración propia con base en datos de la CEPAL (2013).

Aunque las mediciones de las cifras de las tablas 3 y 4 no son completamente similares, sirven para tener una idea de las diferencias existentes entre el acceso a la educación para el estrato de población conformado por niños, niñas y adolescentes en general y el mismo grupo de población con alguna discapacidad. Como se puede observar, de los datos de la Tabla 3, las tasas de escolaridad en el nivel primario son altas para todo el grupo de países referenciados, con tasas iguales o superiores al 94\%. En el nivel secundario, las tasas son un poco más bajas y existen diferencias relevantes entre los países examinados, pues mientras Brasil, Costa Rica, Ecuador y Uruguay tienen tasas de escolaridad secundaria en un rango de 70-80\%, la respectiva de Colombia, México y Panamá es de $60-70 \%$, mientras que la de El Salvador cae hasta 57\%. 
La información que se presenta en la Tabla 4 revela que, en general, para todos los países mencionados, el grupo porcentual de niños, niñas y adolescentes con alguna discapacidad tiene tasas de escolaridad inferiores cuando se les compara con los que no tienen esta condición. No obstante, hay diferencias apreciables entre los respectivos porcentajes de los países. En el caso de Costa Rica, las tasas de escolaridad de los adolescentes entre 13 y 18 años que presentan discapacidad visual, auditiva o del habla están en el rango de 80-90\%, incluso un poco mayor que la matrícula general en el segundo nivel educativo. De la misma manera, esta tasa se mueve en el rango de $70-80 \%$ para estudiantes con discapacidad de aprendizaje, conducta o movilidad, similar a su tasa de matrícula en segundo nivel.

Tres casos similares al de Costa Rica son Brasil y Uruguay, y en menor medida, Panamá, con la salvedad de que para el caso de estas tres naciones los datos están incompletos. Aún con la data incompleta que se expone, estos países también exhiben tasas de inclusión educativa para las personas con discapacidad de edad comprendida entre 13-18 años relativamente altas, incluso iguales o superiores a la tasa de matrícula en general en el nivel secundario.

Ecuador y México tienen tasas promedio de inclusión educativa para estudiantado con alguna discapacidad muy similares a sus respectivas tasas de matrícula en general en su nivel secundario, entre 70-75\% para el caso de Ecuador y 65-70\% para el caso de México. Colombia tiene una tasa de inclusión educativa para las personas con discapacidad entre 1318 años inferior en prácticamente 10 puntos a la que consigue para la matrícula en general para el segundo nivel, pero se hace la salvedad de que la información corresponde al 2005. El de peor desempeño del grupo analizado es El Salvador. De manera similar a su peor desempeño en términos de escolarización en el segundo nivel, con 57\%, en materia de inclusión educativa de las personas con discapacidad entre 13-18 años, solo consigue una tasa de escolarización de 38\%, prácticamente 20 puntos por debajo de la general en el segundo nivel.

Los anteriores datos revelan que en materia de inclusión educativa de las personas con discapacidad todavía la mayoría de las naciones latinoamericanas tienen grandes retos que superar. No solo se necesitan los instrumentos jurídicos ya existentes para garantizar el derecho de las personas con discapacidad, especialmente de niños, niñas y adolescentes, a tener acceso a la educación, a escolarizarse, también se requiere del diseño de planes y 
programas integrales y de la acción y de la coordinación de las instituciones ad hoc para implementar, monitorear y evaluar las políticas públicas que conviertan esa inclusión educativa en una realidad.

\section{Consideraciones finales}

Los países de América Latina han dado importantes pasos en materia jurídica, normativa e institucional en el nivel internacional, regional y nacional para proteger y promover los derechos y la inclusión social de las personas con discapacidad. La ratificación de Convenciones regionales e internacionales, como la "Convención Interamericana para la Eliminación de todas las formas de Discriminación contra las personas con Discapacidad" de la OEA, de 1999, o la "Convención Internacional sobre los Derechos de las Personas con Discapacidad" creada en 2006 y ratificada por todos los países de la región desde 2008 así lo acreditan. Por otra parte, cada país ha creado instrumentos jurídicos y normativos propios para consolidar estos derechos. Hay pues en América Latina una fuerte vinculación entre los cuerpos legales, la capacidad institucional y las políticas públicas dirigidas directa 0 indirectamente a las personas con discapacidad. Todo lo anterior se ha traducido en que los países de la región cuentan con instituciones sociales ministeriales que se abocan a diseñar e implementar las políticas públicas para el alcance del objetivo de proteger y promover los derechos, la inclusión y la plena participación social de las personas con discapacidad.

Estas instituciones, sean centralizadas o descentralizadas, dependientes o gocen de una relativa autonomía, tienen que coordinar y articular sus planes, programas y políticas con otras instituciones de desarrollo social, pues la condición de las personas con discapacidad es transversal, ya que diferentes políticas de desarrollo social, como las políticas de educación, de salud, de transporte, de recreación, tienen efectos en su bienestar. Dado que la discapacidad se distribuye entre diferentes edades, sexo, condición social, los marcos normativos de las políticas sociales institucionales referidos a este grupo poblacional deben tener en cuenta esta transversalidad.

Las personas con discapacidad son susceptibles de ser discriminadas y excluidas de su acceso a la educación, lo cual afecta especialmente a niños, niñas y adolescentes con discapacidad. Por tanto, el reto de las políticas dirigidas a este grupo de población vulnerable es doble. En primer lugar, se trata de asegurar, con las políticas públicas, su derecho a no ser excluidos ni discriminados. Son acciones afirmativas que deben ser prioridad en la 
agenda de planes y programas de las instituciones que les sirven y prestan apoyo. En segundo lugar, se debe buscar la necesaria integración con otros planes, programas y políticas sociales para que la efectividad de la política tenga un impacto sustancial en el mejoramiento de su calidad de vida. En estos términos, no solo se trata de que mediante las leyes y las políticas se asegure el derecho de las personas con discapacidad a acceder a la educación, sino también de que la educación que reciben sea realmente inclusiva, en igualdad de oportunidades y de calidad.

Es aquí donde la tarea no resulta fácil porque, pese a los avances jurídicos y normativos, y los esfuerzos institucionales los resultados prácticos de la acción de las instituciones y de las políticas públicas implementadas aún requieren del monitoreo y de la evaluación rigurosa que señale qué está funcionando y qué no. Aunque existen una serie de casos de políticas exitosas para las personas con discapacidad en toda la región, aún falta mucho por hacer para mejorarlas. Estas deficiencias son palpables en áreas de políticas como el acceso a la educación, especialmente de niños, niñas y adolescentes con alguna discapacidad. Como se constató, analizando la escolarización general y la respectiva para niños, niñas y adolescentes con algún tipo de discapacidad para un grupo de países de la región, la brecha de acceso a la educación para este grupo poblacional es evidente en algunas naciones. Se trata solo de un aspecto cuantitativo, que nada dice del aspecto cualitativo acerca de la calidad de la educación que se les imparte.

Asimismo, las fallas en la política de inclusión educativa necesariamente tendrán efectos desfavorables en otras políticas sociales inclusivas, especialmente en las políticas de formación y de empleo pues el acceso a la educación es previo y se requieren las acciones afirmativas dirigidas a empoderar a las personas con discapacidad, con el fin de que otras políticas, como las de empleo o de inserción laboral productiva sean efectivas. Desde esta perspectiva, surgen una serie de problemáticas alrededor de las políticas públicas dirigidas a las personas con discapacidad, en particular, las orientadas a asegurar el acceso a la educación de niños, niñas y adolescentes que deben considerarse. En este sentido, un aspecto prioritario es el de crear los mecanismos institucionales que permitan generar datos, sistemas de información e indicadores que permitan, en primer lugar, tener un panorama más ajustado y certero acerca de las personas con discapacidad y sus condiciones de vida. En segundo lugar, se requieren sistemas de información que sirvan para la implementación, el monitoreo y evaluación de las políticas, en el entendido de que mayor información y de 
mejor calidad, permitirá conocer cuáles planes, programas y políticas están funcionando de manera efectiva.

Otro aspecto prioritario se presenta en el ámbito interinstitucional. Dado que las políticas sociales se despliegan en la acción de múltiples instituciones y organismos centralizados y descentralizados, dependientes o autónomos, las políticas específicas, focalizadas, dirigidas a las personas con discapacidad, deben de igual manera coordinarse bien e integrarse con las demás políticas sociales, esto para evitar la duplicación del uso de los recursos financieros. Asegurar la eficiencia en el uso de recursos se convierte en una necesidad, ya que normalmente las asignaciones a las instituciones de desarrollo social y las que atienden a las personas con discapacidad no escapan a la volatilidad que, frecuentemente sufren los presupuestos públicos de la región, susceptibles de reducirse 0 eliminarse cuando surgen problemas fiscales.

La conclusión más importante derivada de este análisis es que mejorar los datos, los sistemas de información, la focalización y la articulación institucional de las políticas públicas dirigidas a las personas con discapacidad resulta clave para alcanzar su efectividad. La recomendación más importante es la necesidad de plantear una agenda de investigación universitaria en América Latina en torno a los temas y el fenómeno que impacta en la protección y promoción de los derechos y la inclusión social de las personas con discapacidad. Este ensayo pretende ser un aporte en esa dirección.

\section{Referencias}

Bernal, María E. y Gómez, Mara F. (2012). Innovar en educación: un aporte a la equidad. Documento de Proyecto N LCW.480. Santiago de Chile: CEPAL.

Colamarco, Victoria y Delamonica, Enrique. (2013). Políticas para la inclusión de la infancia con discapacidad (Desafíos, Boletín de la infancia y adolescencia sobre el avance de los Objetivos de Desarrollo del Milenio, $\left.N^{\circ} 15\right)$. Santiago de Chile: CEPAL-UNICEF.

Comisión Económica para América Latina y el Caribe. (2018). Base de Datos y Publicaciones Estadísticas. http://estadisticas.cepal.org/cepalstat/portada.html?idioma=spanish

Comisión Económica para América Latina y el Caribe. (2013). Panorama Social de América Latina 2012. Santiago de Chile: Publicaciones de las Naciones Unidas. 
González, Daniela y Stang, Fernanda. (2014). Las personas con discapacidad en América Latina a 20 años del consenso de El Cairo: la necesidad de información para políticas y programas (Notas de Población № 99). Santiago de Chile: CEPAL.

Lei do Apoio às Pessoas Portadoras de Deficiencia. (1989). Recuperado de https://www.ilo.org/dyn/natlex/docs/ELECTRONIC/57119/97306/F2094854788/BRA571 19.pdf.

Ley de Atención a las Personas con Discapacidad, $N^{\circ}$ 135-96. (1997). Recuperado de http://www.acnur.org/fileadmin/Documentos/BDL/2001/0147.pdf.

Ley de Equiparación de Oportunidades para las personas con Discapacidad, $N^{\circ}$ 8. (2000). Recuperado de https://www.oas.org/dil/esp/Ley de Equiparaci\%C3\%B3n de Oportunidades para las Personas con Discapacidad El Salvador.pdf

Ley de Equiparación de Oportunidades para las Personas con Discapacidad, $N^{\circ}$ 42. (1999). Recuperado de https://www.mire.gob.pa/images/ministerios/leyes-decretos/ley421999.pdf

Ley de Igualdad de Oportunidades para las Personas con Discapacidad, $N^{\circ}$ 7.600. (1996). Publicado en la Gaceta № 102 del 29 de mayo. San José, Costa Rica: Imprenta Nacional.

Ley de los Derechos de las Personas con Discapacidad, $N^{\circ}$ 763. (2011). Recuperado de https://www.ilo.org/dyn/travail/docs/2129/Ley\%20No.\%20763\%20(1).pdf.

Ley de Normas sobre Igualdad de Oportunidades e Inclusión Social de Personas con Discapacidad, $\quad N^{\circ} \quad 20.422 . \quad$ (2010). Recuperado de http://www.sipi.siteal.iipe.unesco.org/sites/default/files/sipi normativa/ley no 20.422 d e 2010.pdf

Ley de protección Integral de personas con Discapacidad, $N^{\circ}$ 18.651. (2010). Recuperado de https://www.bps.gub.uy/bps/file/11976/2/ley-18651.pdf.

Ley para las Personas con Discapacidad. (2006). Recuperado de http://www.medicinalaboraldevenezuela.com.ve/archivo/ley discapacitados.pdf

Ley del Sistema de Protección Integral de los Discapacitados, $N^{\circ}$ 22.431. (1981). Recuperado de http://servicios.infoleg.gob.ar/infoleglnternet/anexos/2000024999/20620/norma.htm.

Ley Estatutaria de Disposiciones para garantizar el pleno ejercicio de los derechos de las personas con discapacidad, No 1.618. (2013). Recuperado de https://discapacidadcolombia.com/phocadownloadpap/LEGISLACION/LEY\%20ESTAT UTARIA\%201618\%20DE\%202013.pdf 
Ley General de la Persona con Discapacidad, $N^{\circ}$ 29.973. (2012). Recuperado de http://www.leyes.congreso.gob.pe/Documentos/Leyes/29973.pdf

Ley General de las personas con Discapacidad. (2005). Recuperado de http://www.salud.gob.mx/unidades/cdi/nom/compi/ley100605.html

Ley General para personas con Discapacidad, $N^{\circ}$ 223. (2012). Recuperado de https://www.migracion.gob.bo/upload/l223.pdf

Ley Orgánica de Discapacidades. (2012). Recuperado de https://oig.cepal.org/sites/default/files/2012 leyorg.dediscapacidades ecu.pdf

Ley Sobre Personas con Discapacidad, $N^{\circ}$ 5-13. (2013). Recuperado de http://www.campanaderechoeducacion.org/sam2014/warehouse/2014/06/Ley-No.-513.pdf.

Meléndez Rojas, Raudin (2018a). Análisis del proceso de implementación de las políticas públicas dirigidas a la educación inclusiva de los adolescentes con discapacidad visual en el Centro Nacional de Educación Helen Keller, períodos 2016-2017 (Tesis para optar por el grado de Doctorado en Ciencias de la Educación). Universidad Católica de Costa Rica, San José, Costa Rica.

Meléndez Rojas, Raudin. (2018b). Educación inclusiva y discapacidad en Costa Rica: una perspectiva desde las políticas públicas. Actualidades Investigativas en Educación, 18(2), mayo-agosto, 1-17. DOI: 10.15517/aie.v18i2.33253

Martínez, Rodrigo y Maldonado Valera, Carlos. (2017). La institucionalidad del desarrollo y la protección social en América Latina y el Caribe: panorama y desafíos. En Rodrigo Martínez (Ed.), Institucionalidad social en América Latina y el Caribe (pp. 53-102). Santiago de Chile: Publicaciones de la CEPAL.

Nazif, José. (2011). Discapacidad y movilidad: interacción de dos políticas públicas en fomento del desarrollo sostenible (Boletín de Facilitación del transporte y el comercio en América Latina y el Caribe $\mathrm{N}^{\circ}$ 298). Unidad de Servicios de Infraestructura de CEPAL. $\quad$ Recuperado https://repositorio.cepal.org/bitstream/handle/11362/36093/1/FAL-298-WEB es.pdf

Organización de Estados Americanos. (1999). Convención Interamericana para la Eliminación de todas las formas de Discriminación contra las personas con Discapacidad. Recuperado de http://www.oas.org/juridico/spanish/tratados/a-65.html

Organización Mundial de la Salud. (2011). Informe Mundial sobre la Discapacidad. Malta: OMS.

Organización de las Naciones Unidas. (2006). Convención sobre los Derechos de las Personas con Discapacidad. Organización de las Naciones Unidas. Recuperado de http://www.un.org/esa/socdev/enable/documents/tccconvs.pdf. 
Programa de Naciones Unidas para el Desarrollo. (2015). Objetivos de Desarrollo Sostenible. Nueva York: Publicaciones de Naciones Unidas.

Red Latinoamericana de Organizaciones No Gubernamentales de Personas con Discapacidad y sus Familias. (2018). Estatuto de RIADIS. Recuperado de http://www.riadis.org/estatuto/

Rico, María y Robles, Claudia. (2017). El cuidado, pilar de la protección social: derechos, políticas e institucionalidad en América Latina. En Rodrigo Martínez (Ed.), Institucionalidad social en América Latina y el Caribe (pp. 219-247). Santiago de Chile: Publicaciones de la CEPAL.

Rodríguez, Viviana y Salazar, Juan Pablo. (2011). Calidad en el servicio al cliente: una visión diferente. San José, Costa Rica: Guilá Imprenta y Litografía.

Stang, María F. (2011). Las personas con discapacidad en América Latina: del reconocimiento jurídico a la desigualdad real. Serie Población y Desarrollo $N^{\circ} 103$, Abril. Santiago de Chile: CEPAL.

Ullmann, Heidi. (2017). Discapacidad y políticas públicas: avances y desafíos institucionales en América Latina. En Rodrigo Martínez (Ed.), Institucionalidad social en América Latina y el Caribe (pp. 275-299). Santiago de Chile: Publicaciones de la CEPAL. 
Revista indizada en

scip/o redalyc. latindex

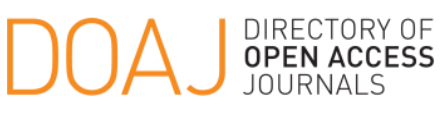

Distribuida en las bases de datos:

- Dialnet

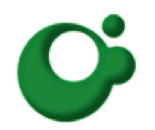
SHERPA/RøMEO REDIB

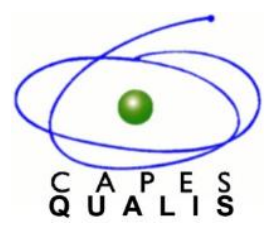

MIAR 